\title{
Mathematical Modeling and Analysis of Mitochondrial Retrograde Signaling Dynamics
}

\author{
Shao-Ting Chiu ${ }^{1}$, Wen-Wei Tseng ${ }^{1}$, An-Chi Wei ${ }^{1 *}$ \\ ${ }^{1}$ Graduate Institute of Biomedical Electronics and Bioinformatics, National \\ Taiwan University, Taipei, Taiwan \\ *acwei86@ntu.edu.tw
}

\begin{abstract}
Mitochondria, semi-autonomous eukaryotic organelles, participate in energy production and metabolism, making mitochondrial quality control crucial. As most mitochondrial proteins are encoded by nuclear genes, quality control depends on proper mitochondria-nucleus communication, designated mitochondrial retrograde signaling. Early studies focused on retrograde signaling participants and specific gene knockouts. However, mitochondrial signal modulation remains elusive. Using yeast, we simulated signal propagation following mitochondrial damage and proposed a mathematical model based ordinary differential equations. Mitochondrial retrograde signaling decisions were described using a Boolean model. Dynamics were analyzed through an ordinary differential equation-based model and extended to evaluate the model response to noisy damage signals. Simulation revealed localized protein concentration dynamics, including waveforms, frequency response, and robustness under noise. Retrograde signaling is bistable with three localized steady states, and increased damage compromises robustness. We elucidated mitochondrial retrograde signaling, thus providing a basis for drug design against yeast and fungi.
\end{abstract}

\section{Author Summary}

The yeast RTG pathway regulates mitochondrial metabolism and mitochondrial quality through passing mitochondrial signal to the nucleus to modulate gene expressions. Using microscopic data of RTG proteins translocation from cytosol to nucleus, the parameters were found by fitting 16 knockout conditions simulated from the proposed differential equation-based model. Further, through dose response, ultrasensitivity, frequency response and noise interference, we demonstrated a switch-like property of RTG pathway activation, a capacity charging property of RTG protein translocation, a low pass filter property of signals in different frequencies, and noise amplification due to mitochondrial signal. The proposed mathematical model enables us to understand the dynamics and mechanisms in mitochondrial retrograde signaling in yeast and provides potential antifungal treatment strategies. 


\section{Introduction}

Mitochondria serve as the cells' powerhouse, utilizing the established proton motive force to generate the high-energy molecule, ATP, thus facilitating various cellular processes 1 . The analogy of a simple electrical circuit model is a convenient way to describe the interactions between ion dynamics and energetics in the complex mitochondrial system $[2,3]$. Mitochondria regulate various cellular mechanisms under normal physiological conditions, as well as in disease, including ATP generation, regulation of signal transduction, immune responses, and cell apoptosis [1]. Therefore, mitochondria serve as an essential regulatory metabolic hub 4 .

However, their extensive role within the cell renders mitochondria prone to be damaged [5,6]. Mitochondrial quality control is therefore essential for cell viability, which relies on mitochondria-driven energy production [1], lipid synthesis [7, and amino acid metabolism [8]. However, energy production via the electron transport chain results in an oxidative stress burden [9]. Unlike other membrane-bound organelles, which rely solely on protein products from the nuclear genome, mitochondria possess their own genome, encoding dedicated enzymes and repair systems; however, they remain semi-autonomous organelles, as most mitochondria protein-encoding genes are translocated into the nucleus [10]. In fact, in budding yeast, only 35 of the 1,000 mitochondrial genes are located in the mitochondrial genome, representing a minor fraction of the metabolic system [11]. Therefore, mitochondria require various products from nucleus genome to maintain functionality. The interaction between mitochondria and the nucleus is a bidirectional process 4,12 . Once mitochondria are damaged, their status is reported to the nucleus via signaling factors or metabolic pathways, enabling the cell to coordinate mitochondrial biogenesis. Furthermore, mitochondrial quality control depends on mitochondrial feedback, referred to as mitochondrial retrograde signaling [5, 12, 14].

The most well-characterized mitochondrial RTG signaling cascade is that in budding yeast 12,15,18, which gets triggered when mitochondrial respiratory function is compromised [19], causing a shift in carbohydrate and nitrogen metabolism [20]. CIT2, one of the regulated genes, encodes a peroxisomal isoform of citrate syntheses involved in the glyoxylate cycle, thereby bypassing the TCA cycle. Thus, the upregulation of CIT2 expression allows for more efficient usage of carbon sources when mitochondria are damaged. This RTG pathway was first characterized by investigating various yeast gene deletion strains. For instance, in a study by Sekito et al. [18], RTG genes were independently knocked out, and green fluorescent protein (GFP) was employed to localize the RTG proteins, revealing details of the mitochondria-to-nucleus communication system. Furthermore, the localization of RTG proteins was compared among deletion strains, as well as strains with dysfunctional mitochondria, such as $\rho^{0}$ cells, which lack a mitochondrial genome.

The RTG pathway is initiated by the activation of Rtg2p, a cytoplasmic 
sensor of mitochondrial damage with an ATP-binding domain similar to the Hsp70/actin/sugar kinase superfamily. Rtg2p is activated by the loss of mitochondrial membrane potential or the ATP/ADP ratio [12,20, 21], which results in the degradation of Bmhp/Mksp heterodimer that inhibits the Rtg3p activation [17,21]. Rtg1p/3p are are basic helix-loop-helix-leucine zipper (bHLH/Zip) transcription factors that induce the retrograde response via nucleus translocation with nucleus localization signal (NLS) and binding to the promoter R box region of RTG response genes to initiate transcription [22].

Without Rtg2p sensing mitochondrial status, Rtg3p and Rtg1p localization occurs independently of mitochondrial damage because of constant inhibition of Mksp. Rtg3p and Rtg1p remain cytoplasmic in wild type and dysfunctional mitochondria ( $\rho^{0}$ strain) [18]. In addition, Rtg1p regulates the translocation of Rtg3p. Although Rtg1p participates in the activation of the retrograde response, it retains Rtg3p in the cytoplasm in the absence of mitochondrial damage. In contrast, Rtg3p accumulates in the nucleus regardless of mitochondrial status in Rtg1p knockout mutants, while the opposite is not true. Initiation of retrograde response signaling requires nuclear accumulation of both Rtg3p and Rtg1p like the AND logic gate [18,23,24. Therefore, Rtg1p acts as a positive regulator of retrograde response genes, as well as a negative regulator that retains Rtg3p in the cytoplasm in the absence of mitochondrial damage.

Cells usually harbor a number of mitochondria that share the same retrograde signaling pathway, resulting in a multiplexing issue first described in telecommunications [25]. Sharing signaling channels may lead to unwanted crosstalk. Further, biochemical pathway noise restricts the cells' ability to receive information from their organelles. In eukaryotic cells, such as budding yeast, mitochondria interact with the nucleus via the same biochemical channels, and the consequent multiplexing issue represents a barrier to assessing the health state of each mitochondrion. How biological systems manage multiplex communication networks during the mitochondrial RTG response remains unclear.

To investigate the communication properties of mitochondrial retrograde signaling, we have developed a differential equation-based model to simulate the process. The mitochondrial status and deleted genes are defined as input, whereas translocation of Rtg1p/Rtg3p is the defined output. These interactions were previously observed using immunofluorescence microscopy [18, 32, and subsequently summarized in a Boolean table. The parameters were fitted with the Boolean relationship using Monte Carlo simulation. A parameter set was verified by solving the steady states under each observed genotype. Collectively, our mathematical model interprets the dynamics of mitochondrial retrograde signaling. Furthermore, the proposed mathematical model also has potential applications in research on antifungal drugs and aging. 


\section{Results}

\section{A differential equation-based model of mitochondrial retrograde signaling}

As a channel of communication between mitochondria and the nucleus, RTG signaling is well regulated and fine-tuned for a proper response to mitochondrial damage signals. The dynamics and interactions of Rtg1p, Rtg2p, Rtg3p, Msk1p, and Bmhp can be described using ordinary differential equations (ODE) and biochemical reaction network. The first part of RTG signaling is the activation layer, during which the mitochondrial damage signal activates Rtg2p. Then, the signal is transmitted to the modulation layer where Rtg1/3p translocate into the nucleus and initiate transcription (Fig 1 p). For simplicity, the chemical interactions among retrograde proteins and the translocation of $\mathrm{Rtg} 1 / 3 \mathrm{p}$ are assumed to obey the law of mass action, and the total concentration of each protein was regarded as conserved. To connect retrograde proteins with mitochondrial damage, the activation of Rtg2p was modeled using the Hill equation with the sum of mitochondrial damage. Furthermore, proteins with activation states or locations are defined separately, leading to a total of 17 protein variables and 24 kinetic coefficients (Table S3).

To identify the parameter set that would make the model compatible with the experimental observations 18,32,34, a Monte Carlo simulation method was used to perform the parameter exploration. First, to leverage the microscopic data 18, 32, the translocation of Rtg1/3p and mitochondrial states were summarized in a Boolean model with 17 genetic backgrounds, including the combination of RTG gene knockouts (Table 1). Second, the relative protein concentrations of Bmhp and Rtg1/2/3p were determined based on the gene expression 35 and quantitative western blot analyses 34. (Fig S1). Third, a random search method was applied to explore the parameter set. Finally, the steady states of the model were compared to the Boolean model (Fig 2 p). A parameter set was regarded as valid when the steady states fulfilled the observations of 17 knockout conditions.

\section{The ultrasensitivity of Bmh/Mksp degradation causes a switch-like response of $\mathrm{Rtg} 1 / 3 \mathrm{p}$ translocation to mitochondrial damage}

Nuclear accumulation of Rtg1p and Rtg3p activates the retrograde response. To understand the mechanism by which information is propagated from the upstream Bmh/Mksp inhibitor to Rtg1/3p translocation, a sigmoid signal of mitochondrial damage was applied to simulate the transition of RTG proteins. The step response revealed sophisticated details on RTG protein dynamics induced by the fast sigmoid transition of the damage signal (Fig 10). The degradation of Bmh/Mksp indicated an ultrasensitive response to the input signal. However, the translocation of Rtg1p and Rtg3p was relatively smoother and delayed. The delayed stimulation of Rtg1p and Rtg3p may be caused by the slow Rtg3p autoactivation rate. Besides, the step responses overshoot pattern 
Fig 1. Mitochondrial retrograde signaling pathway model.

(a) The mitochondria-to-nucleus communication circuit. Retrograde signaling is divided into the activation layer and modulation layer. Retrograde proteins (Bmhp, Mksp, and Rtg1/2/3p) are classified into an active (-act) and inactive state (-ina). Cellular compartments are divided based on the nucleus (-nuc) and cytosol $(-c y t)$. The arrow represents the binding of two proteins, transformation of active states, or translocation between nucleus and cytosol, with the kinetic coefficient labeled besides it. The input $\mathrm{s}$ is defined as the summation of mitochondrial damage signals. Rtg2p is activated once the mitochondrion loses its membrane potential, or the ATP concentration decreases. The active form of Rtg2p suppresses the formation of the Mks/bmhp complex by competitive binding with Mksp, resulting in dephosphorylation of Rtg3p and translocates to the nucleus accompanied by Rtg1p. The Rtg1p-Rtg3p complex eventually triggers the retrograde response and upregulates CIT2 expression. (b) Step responses of Bmh/Mksp, Rtg1 $\mathrm{p}_{n}$ and $\mathrm{Rtg} 3 \mathrm{p}_{n}$ showing switch-like responses of Bmh/Mksp, capacity-charging curve with a time delay for $\operatorname{Rtg} 1 \mathrm{p}_{n}$ and delayed two-staged rise with a sharp transition at the beginning, for Rtg3 $\mathrm{p}_{n}$. (c) The sinusoidal inputs of $1.6 \times 10^{-2} \mathrm{~Hz}$ response reveals RTG signaling pathway as a low-pass filter. The mitochondrial damage signal (thin gray line), labeled as the input with quantity shown on the right y-axis. The concentration of RTG proteins (thick blue line), regarded as outputs with quantities labeled on the left $\mathrm{y}$-axis. To normalize the response, the mass fraction is defined as the ratio of the concentration of a given protein set to its total concentration. Nucleus accumulation is described by the ratio of nucleus concentration to total protein concentration. 


\begin{tabular}{ccccccc}
\hline Rtg1p & Rtg2p & Rtg3p & Mito-damage & Mks & GFP-labelled protein & Nucleus Accumulation \\
\hline 0 & 0 & 1 & 0 & 1 & Rtg3-GFP & N/A \\
0 & 0 & 1 & 1 & 1 & Rtg3-GFP & 1 \\
0 & 1 & 1 & 0 & 1 & Rtg3-GFP & 1 \\
0 & 1 & 1 & 1 & 1 & Rtg3-GFP & 1 \\
1 & 0 & 1 & 0 & 1 & Rtg3-GFP & 0 \\
1 & 0 & 1 & 1 & 1 & Rtg3-GFP & 0 \\
1 & 1 & 1 & 0 & 1 & Rtg3-GFP & 0 \\
1 & 1 & 1 & 1 & 1 & Rtg3-GFP & 1 \\
1 & 0 & 0 & 0 & 1 & Rtg1-GFP & N/A \\
1 & 0 & 0 & 1 & 1 & Rtg1-GFP & 0 \\
1 & 1 & 0 & 0 & 1 & Rtg1-GFP & 0 \\
1 & 1 & 0 & 1 & 1 & Rtg1-GFP & 0 \\
1 & 0 & 1 & 0 & 1 & Rtg1-GFP & 0 \\
1 & 0 & 1 & 1 & 1 & Rtg1-GFP & 0 \\
1 & 1 & 1 & 0 & 1 & Rtg1-GFP & 1 \\
1 & 1 & 1 & 1 & 1 & Rtg1-GFP & 1 \\
1 & 0 & 1 & 0 & 0 & Rtg3-GFP & 1 \\
1 & 1 & 1 & 0 & 0 & Rtg3-GFP & 1 \\
1 & 0 & 1 & 0 & 0 & Rtg1-GFP & 1 \\
1 & 1 & 1 & 0 & 0 & Rtg1-GFP & \\
\hline
\end{tabular}

Table 1. The Boolean model of mitochondrial retrograde signaling. The existence of RTG proteins and mitochondrial damage signal is described in binary quantity based on the experimental observations of the sub-cellular localization of GFP-tagged-RTG proteins in wild type and $\operatorname{rtg} \Delta$ mutant derivatives of $\rho_{+}$and $\rho_{0}$ cells [18]. The 0 represents deleted protein or absence of mitochondrial damage, and 1 represents the opposite phenomena. Untested conditions are designated as N/A. 
Fig 2. Boolean decision in RTG signaling pathway.

(a) Logic gate analog for the RTG signaling pathway Rtg1p(left) and Rtg3p(right). Mitochondrial damage and the existence of Rtg1p, Rtg2p, and Rtg3p determined the translocation of Rtg1p into nucleus through a series of logic gate decisions (Table S2). The input is defined as 0 with knockout protein or absence of mitochondrial damage, and 1 represents the existence of a protein or mitochondrial damage. On the other hand, the output is defined as 1 with nucleus accumulation and 0 means no accumulation event. The solid line represents logic gate decision for nucleus accumulation, and the dotted line represents biological process that leads to retrograde response. (b) Boolean transformation of Rtg proteins nucleus translocation in healthy and damaged mitochondria. Simulated distribution of GFP-labeled Rtg1p under multiple conditions was compared to the Boolean model. The steady states of GFP proteins are listed by the cell icon labeled with nucleus (inner circle) and cytoplasmic (outer circle) concentration in arbitrary units. Localized concentrations are described as the summation of GFP-labeled proteins, including heterodimers. The relative quantity indicates the localized concentration-to-summation ratio, from 0 (gray) to 1 (green). To verify the result, simulated distributions are compared with the qualitative data of $\Delta r t g 1 / \Delta r t g 2 / \Delta r t g 3[18$ and $\Delta m k s[21]$. Nuclear accumulation is regarded as nuclear-to-cytoplasmic ratio is greater than 1.5. Simulation distribution of GFP-labeled Rtg1p(left) and GFP-labeled Rtg3p(right).

was identified in the particular forms of Rtg1p and Rtg3p, while summation canceled this effect (Fig S2a).

Two forces control the nuclear translocation of Rtg3p, namely autoactivation of the NLS and degradation of the cytoplasmic Rtg1/3p heterodimer, releasing extra Rtg3p monomers that permeate the nuclear membrane. When the mitochondrial damage signal degrades Bmh/Mksp via Rtg2p, the autoactivation of Rtg3p turns on the NLS and increases the influx kinetic coefficient.

Furthermore, the overshoot of activated Rtg3p concentration causes the decline observed in the early response (Fig S2a). The decreased cytoplasmic Rtg3p concentration causes further degradation of the Rtg1/3p heterodimer, releasing more free-Rtg3p. Autoactivation of the Rtg3p NLS contributes to the sharp increase in Rtg3p nuclear concentration shortly after the damage signal. Once autoactivation reaches a steady-state, free Rtg3p is conserved and translocated to the nucleus by simple diffusion. Hence, the transition of Rtg3p becomes smoother in the second stage, leading to a capacity-charging curve at the end (Fig $\mathrm{S} 2 \mathrm{~b})$. In addition to Rtg3p, the translocation of Rtg1p also results from degradation of the cytoplasmic Rtg1/3p heterodimer and simple diffusion. When Rtg1p is absent or negligible, simple diffusion causes Rtg3p to accumulate in the nucleus independently of the damage signal. The role of Rtg1p is to retain Rtg3p in the cytoplasm, however, the anchoring force is insufficient to retain activated Rtg3p [18]. The current simulation supports previous observations and provides details regarding the ultrasensitivity of Bmh/Mksp 
degradation, the two-staged transition of Rtg3p, and the capacity-charging curve of Rtg1p in response to mitochondrial damage signals.

151

152

\section{Competitive binding to Mksp between Bmhp and Rtg2p contributes to the ultrasensitivity of Bmh/Mksp degradation}

The ultrasensitivity of Bmh/Mksp degradation is indicated by the phase plot with mitochondrial damage as input (Fig 3). Although the Hill function models this process with the dissociation constant of input $\left(k_{s d}\right)$, the phase plot of Rtg2p reveals a two-staged sigmoid transition with two thresholds significantly below $k_{s d}$, and with higher stiffness on the first one. The degradation of Bmh/Mksp occurs at the first transition, wherein the threshold for sensing mitochondrial damage is lower than that of the input Hill function. Therefore, the ultrasensitivity of Bmh/Mksp is independent of the Hill input model (Fig S3) and is caused by the competitive binding between activated Rtg2p and Bmhp.

To quantify the sensitivity, the relative amplification method [36], a quantitative approach for estimating the Hill coefficient with basal activation, was applied. As shown in Fig 3c, the response of inactive Rtg2p (Rtg2p $\left.{ }^{i n a}\right)$ is with lower Hill coefficients for both transitions compared to the input locally and globally (Fig 3p, left). Meanwhile, the degradation of the Bmh/Mksp heterodimer occurs at the first transition of Rtg2 $\mathrm{p}^{\text {ina }}$, with a higher Hill coefficient, which was lower than that of the input (Fig $3 \mathrm{k}$, right). Via the relative amplification method, we estimated the Hill coefficient numerically. Despite knowing the quantity of the Hill coefficient, the transition of Bmh/Mksp occurred far below the dissociation constant $k_{s d}$, which is unlikely to be caused by the input Hill function, leading to questions regarding the source of its ultrasensitivity. To elucidate the underlying mechanism, we analyzed the Rtg2p-Mksp-Bmhp motif by solving the steady states algebraically. The exact analytical solution revealed that the transitions of inactivated Rtg2p stem from the molecular titration. Furthermore, the competitive binding between Rtg2p and Bmhp with Mksp forms a binary switch that could process the analog input signal into digital information.

\section{Frequency modulation of mitochondrial retrograde signaling}

In addition to the dose response, we further investigated the frequency modulation of the RTG signaling. Bmh/Mksp heterodimer, and nuclear Rtg1p, and Rtg3p in response to a sinusoidal damage signal of multiple frequencies exhibited low-pass filter behavior (Fig 4 and Fig [S4). The recovery rate of $\mathrm{Bmh} / \mathrm{Mksp}$ synthesis was slower than its degradation, leading to a sign-sensitive delay that keeps the switch on after damage is detected [37], while Bmh/Mksp can be slowly synthesized for a long period of approximately $3 \times 10^{3} \mathrm{~s}$ compared to the transition of less than $10 \mathrm{~s}$. Although the concentration of Bmh/Mksp recovers slowly after stimulation, Rtg1p and Rtg3p are still able to convey input signal dynamics. At a low frequency, both reach high and low saturation states in a sinusoidal input cycle and produce a square wave pattern. As the frequency becomes high, the amplitude of output diminishes with drifting, indicating that 
Fig 3. Ultrasensitivity of the Bmh/Mksp degradation results from molecular titration.

(a) Scheme of the competitive binding between Rtg2p and Bmhp with Mksp. The forward reactions are labeled with thick arrows, and reverse reactions are labeled with dashed arrows. (b) Phase plots. The input signal is defined within the interval between 0 and 1 , and the corresponding steady states are plotted separately with the parameters: $n=7, k_{s d}=0.96, k_{s v}=11.67, k_{2 i}=4.94$, $k_{2 m i}=0.043, k_{2 m}=1604.15, k_{b m i}=2.413, k_{b m}=0.059$ in arbitrary unit.(c) Relative amplification approach of Rtg2p ${ }^{i n a}$ and Bmh/Mksp in response to mitochondrial damage. The activated fraction is converted from the input signal $s$ normalized by its range [36]; the response coefficient is the normalized sensitivity of output with respect to the input signal [57]. The transformed responses of Rtg2p ${ }^{i n a}$ and Bmh/Mksp (thick blue line) are compared via the Michaelis-Menten reaction with a Hill coefficient equal to one, described as a linear function (dashed line) [36]. $n_{\text {global }}$ represents global Hill coefficient; $n_{1}$ and $n_{2}$ are local Hill coefficients in the first and second transition of Rtg2 $\mathrm{p}^{i n a}$.

mitochondrial damage signal is low-pass filtered by retrograde signaling.

Moreover, the delay of RTG translocation may contribute to the instability and compromise robustness. To investigate the feedback instability of mitochondrial retrograde signaling, we further derived a Bode plot of the retrograde response in the stationary stage ( $\mathrm{Fig} \mathrm{S} 4$ ). In the frequency response of $\mathrm{Bmh} / \mathrm{Mksp}$, the gain decreased in two stages separated by $10^{-0.5} \mathrm{~Hz}$. The gain of the high-frequency stage decreased more rapidly than in low frequency stage. On the other hand, while the phase delay increased significantly in the high-frequency region. The cutoff frequency of nuclear Rtg1p and Rtg3p was approximately $10^{-2} \mathrm{~Hz}$ as low-pass filters. For nuclear Rtg3p, there was a second cutoff frequency at approximately $10^{-0.5} \mathrm{~Hz}$ with a more rigid downhill slope. Finally, the Nyquist stability theorem was applied to identify the closed-loop stability of mitochondrial retrograde signaling [38]. Despite the phase delay, retrograde signaling remained stable in response to the damage signal. The phase and gain margin derived from the Bode plots can further indicate the stability tolerance to delay caused by the molecular communication like protein translocation. The low-pass filtering can remove the perturbation that is usually in high frequency (Fig 4 a) 39 .

\section{The intensity of the mitochondrial damage signal decreases the robustness of $\mathrm{Rtg} 1 / 3 \mathrm{p}$ translocation}

Though the dose-response curve describes the communication between mitochondria and nucleus in a deterministic point of view, the molecular noise causes uncertainty and further limits the information conveyed to the nucleus 62,63$]$. To understand how noise influences robustness along with the damage signal, the ODE model was further extended to Chemical Langevin Equations 64. The proposed deterministic model is incorporated with the random chemical reaction, and the potential landscape of nuclear Rtg1/Rtg3p 
Fig 4. Frequency modulation of RTG pathway.

(a) Multiplex processing of RTG signaling. The multiplexed signals from two individual mitochondria are low-pass filtered and delayed with respect to the frequency. The two inputs are set at $0.05 \mathrm{rad} / \mathrm{s}$ (source A) and $0.5 \mathrm{rad} / \mathrm{s}$ (source B) with the same phase. The overall effect on Rtg2p activation is summed with equal weights, and the combined signal is further modulated by the RTG pathway and detected by nucleus Rtg1/3p as the output. (b) Frequency response to mitochondrial damage, Rtg3p as an example. (Left) Gain plot. Low-pass filtering is observed by screening the input frequency from $10^{-3}$ to 1 $\mathrm{Hz}$ with sinusoidal wave. The gain of nucleus Rtg3p is defined as the ratio of output to input amplitude measured by the peak-to-peak distance. (Middle) Phase plot. The delay of output is increased by the input frequency. With the time deviation between the peaks of output and input, the delayed Rtg3p signal is described by degrees in respect of the input frequency. (Right) The Nyquist plot, combined with the gain and phase plot, reveals the closed-loop stability of the proposed model. The radius represents the gain $(\mathrm{dB})$, and the angle labels the degree of phase. The red dot represents the critical point $(-1+0 i)$. The proposed model is stable if the Nyquist curve excludes the critical point.

was derived by screening the damage input with ensemble simulations (Fig $5 \quad 221$ and Method Details). Along with the damage signal, the sigmoid curve of the ${ }_{222}$ potential landscape was consistent with the deterministic results of Rtg1/Rtg3p 223 (Fig 5b). Further, three conjugated steady states were identified in response to a 224 constant damage (Fig 5). A steady state represents the Rtg3p distribution that ${ }_{225}$ is in high probability. The conjugated steady states became distanced as the $\quad 226$ input signal increased, indicating that noise was amplified and caused an 227 increased bandwidth, leading to a compromised signal-to-noise ratio. Notably, ${ }_{228}$ three conjugated steady states were further investigated by measuring the $\quad 229$ output distribution for a given input (Fig 5). The three conjugated steady states 230 were identified by the peak of the probability, which represented the high likelihood of the Rtg1/Rtg3p concentration in a given input. The conjugated steady states were located within the bandwidth of the probability density function (PDF), and the length of bandwidth was increased by the signal. This result suggested that mitochondrial retrograde signaling was not only bistable but also a toggle-switch with multiple locally conjugated stable steady states (Fig 5;). In addition, the uncertainty of the output was worsened by a high input signal, which might compromise information relayed between mitochondria and the nucleus when the former continues to send high-intensity messages. 
Fig 5. Robustness of $\mathrm{Rtg} 1 / 3 \mathrm{p}$ translocation in response to mitochondrial damage signal.

(a) The potential landscape of the nuclear Rtg1/Rtg3p response to mitochondrial damage signals. The sigmoid transition is identified in Fig S3a however, the bandwidth (the dark area in the vertical section) increases in parallel to the damage signal. The damage signal is sampled in 100 grids and assigned to the Chemical Langevin Model in order to simulate the stochastic process. Every combination of parameters is applied with 100-time length (arbitrary unit) with automatic time step. The y-axis is limited within the range of 0 and the maximum concentration of Rtg3p. A heatmap shows the probability with a color bar on the right side. (See Method Details.) (b) The probability density function (PDF) with respect to Rtg1/Rtg3p influenced by damage signal. The distribution is simulated by setting the input signal as 0 (blue, minimal input), 0.45 (orange, intermediate input), and 1 (green, maximum input). The PDF is measured by time series simulation and estimates the probability via kernel density estimation

\section{Discussion}

The RTG retrograde signaling pathway is as an important mechanism for mitochondrial quality control as it serves as both a sensor and a reporter of the mitochondrial health status 13. Quantitative and systemic analysis of this major communication channel will help us elucidate the crosstalk between mitochondria and the nucleus, which maintains cellular function under physiological and pathological conditions. Herein, we constructed a novel mathematical model of mitochondrial retrograde signaling in yeast, applying microscopic data of the RTG pathway knockout experiments with gene and protein expression data for model validation. The differential equation-based model provides a comprehensive approach for studying the dynamic response of mitochondrial retrograde signaling, which has been experimentally challenging due to the lack of means for precise mitochondrial quality manipulation in real time. Retrograde signaling dynamics were observed after introducing the input signal with step and sinusoidal waveforms. Moreover, we applied an analytical solution to identify the source of ultrasensitivity in the degradation of the Bmh/Mksp heterodimer.

The switch-like behavior of the yeast RTG pathway was previously described by 12 . We validated switch-like behavior in this retrograde signaling model (Fig 1) through mathematical formulation and numerical simulation. We observed that switch-like behavior resulted from the competitive binding between active Rtg2p and Bmhp. Besides, the effect of molecular titration triggered a two-staged transition in inactive Rtg2p. Ultrasensitivity is typical in cellular communication, allowing cells to remove noise and amplify input signals 40 42. Rtg2p senses mitochondrial quality through the modulation of Bmhp and Mksp, facilitating detection via competitive binding. The "all-or-none" response can further filter the fluctuation and adjust to the unpredictable environment. 
The current study further elaborates on the properties of signal modulation 268 via Rtg1/3p translocation following static and sinusoidal damage signals. Rtg1p ${ }_{269}$ acts as both a positive and negative regulator [18, retaining the transcription $\quad 270$ factor Rtg3p in the cytoplasm. This considerably constrains the valid parameter ${ }_{271}$ set. Further mechanistic details were identified by the proposed model: the two-staged transition of Rtg3p translocation in step response and the sign-sensitive delay of Rtg3p with a short ON delay in oscillation signal.

Without NLS modification, Rtg1p is required to retain Rtg3p in the cytoplasm when a mitochondrial damage signal is absent, which is one of the criteria of our model. Rtg1p exerts an anchoring effect on Rtg3p by forming a heterodimer, which may not permeate through the nuclear membrane. The translocation of Rtg1p is not affected by the sign of the input change and reveals a simple diffusion process with a capacity-charging pattern. The frequency response further revealed the low-pass filtering of the RTG pathway, which can reject high-frequency fluctuations and retain the overall information of the input signal for requesting nuclear supply (Fig 6).

In the current study, we demonstrated feedback stability of RTG signaling using Bode and Nyquist diagrams [38]; the communication between mitochondria and the nucleus is a two-way process [43]. The phase and gain margin in the Bode plot determined the stable region when cascading with these functional units under mitochondrial oscillation.

Our model also elucidates how the RTG pathway processes the input signal as a low-pass filter, reporting status to the nuclear genome, which may, in turn, support the synchronization of mitochondrial membrane potential in yeast 44-46. and insulin secretion from pancreatic cells 47. Moreover, mitochondria undergo energization cycles driven by the ultradian clock, contributing to respiratory oscillations of mitochondria [44 46]. These oscillations may be a source of reactive oxygen species generated by mitochondria [45], and further interfere the retrograde signaling with sinusoidal input.

The mitochondrion acts as a biochemical signaling hub in eukaryotic cells 48]. Apart from energy production, this semi-autonomous organelle also participates in various metabolic reactions related to aging [49 51], cellular communication [4, 47] and cell viability 26,30 . The RTG response facilitates the adaptation of eukaryotic organisms to unfavorable factors 26, 27. In yeast, the key role of RTG signaling is the regulation of mitochondrial function and metabolic reprogramming, including the maintenance of intracellular glutamate supplies 27,28. Studies have reported the association between the retrograde response and drug resistance in yeast 27, 29, 30. For example, Rtg3p has been reported as essential for antifungal drug tolerance associated with the formation of the fungal plasma membrane, which contains ergosterol [31]. The $\Delta$ rtg3 mutant of pathogenic yeast, Candida albicans, exhibits compromised biofilm formation and epithelial cell adherence, resulting in reduced infectivity of nematode modelCaenorhabditis elegans [29]. Moreover, in Saccharomyces cerevisiae, pleiotropic drug resistance is activated by RTG signaling, inducing the transcription of pleiotropic drug resistance genes [27. RTG signaling is also 1 272 273 274 275 276 277 278 
associated with aging, highlighting yeast as a model organism for the study of mitochondrial quality control and its influences on lifespan $[22$.

Retrograde signaling is connected to glutamate synthesis, mTOR signaling, and other feedback circuits $5,13,52$. The RTG pathway is a major factor in cellular adaptation, serving a cytoprotective role via metabolic reprogramming [6,27]. The robustness of retrograde signaling is essential for adjusting to unfavorable conditions [26]. For instance, RTG signaling contributes to drug resistance in pathogenic yeast, such as C. albicans, which is responsible for approximately $80 \%$ of major systemic fungal infections [27, 53]. Various antifungal drugs target mitochondria-related amino acid and ergosterol metabolism 54,55, and the RTG pathway enables pathogenic yeast to overcome therapeutic agents by sensing the loss of mitochondrial functionality and subsequently reprogramming their metabolism [27, 31, 56. Hence, the yeast retrograde signaling system represents a target for the development of novel antifungal drugs [27]. However, how yeast senses mitochondrial stress via the RTG pathway in a dynamic environment remains unclear.

In this study, we applied mathematical modeling to unravel the dynamics of RTG signaling from a control system point of view. We further identified the threshold of the RTG response, which can be used for determining the optimal dosages for the elimination of pathogenic yeast without triggering RTG signaling. The frequency response reveals how yeast manages to resist fluctuation via low-pass filtering of the RTG pathway, as well as the hysteresis effect, which maintains retrograde signaling as the damage signal fluctuates, indicating that persistent treatment with a concentration slightly below the RTG response threshold is an optimal strategy against pathogenic yeast. Therefore, comprehensively understanding the qualitative properties of retrograde signaling may shed light on signaling mechanisms and provide potential therapeutic strategies.

\section{Fig 6. Mitochondria retrograde circuit.}

(a) Mitochondrial retrograde signaling facilitates communication between the mitochondria and nucleus. The retrograde molecular circuit transduces the signal through the activation layer and the modulation layer to sense and to report the signal to the nucleus. (b) Retrograde signaling circuit was switched on by a mitochondrial damage signal, while a low-pass filter maintained the robustness of the pathway.

\section{Acknowledgment}

The work was supported by the Ministry of Science and Technology in Taiwan ( 343 MOST-109-2636-B- 002 -001 and MOST-110-2636-B- 002 -001 grants to AW). ${ }_{344}$ 


\section{Data and Code Availability}

The source code is available at https://github.com/NTUMitoLab/MitoRetroDynamics. Julia toolbox developed for potential map generator, ultrasensitivity analysis, and steady state explorer are also available at GitHub.

\section{Method Details}

\section{Boolean model of mitochondrial retrograde signaling}

To summarize the systematic behavior of mitochondrial retrograde signaling, a Boolean model was established to discretize the mechanism of protein translocation associated with mitochondrial damage. In this model, The population of $N$ mitochondria is indexed into a vector $\overrightarrow{M T}(\mathrm{Eq} .11$.

$$
\overrightarrow{M T}:=\left[M T_{1}, \ldots, M T_{N}\right]
$$

Though it is known that damaged mitochondria activate Rtg2p and promote the dephosphorylation of Rtg3p by decreasing the Bmh/Mksp heterodimer [16, 18, it is unclear how Rtg2p senses mitochondrial dysfunction. Therefore, we assume mitochondria activates Rtg2p simultaneously under Michaelis-Menten kinetics, and the activation rate of Rtg2p is influenced by the weighted linear summation of mitochondrial damage signals $(S)$ with their volume(Eq. 2).

$$
S=\sum_{i=1}^{N} \text { Volume }_{M T_{i}} \times \text { Damaged }_{M T_{i}}
$$

In the Boolean model, the signal $(S)$ is set to 0 for the healthy mitochondrial population and to 1 when mitochondria are damaged, as in $\rho^{0}$ yeast cells which have no mitochondrial DNA. These genetically damaged mitochondria have no respiratory functionality and constitutively activate retrograde signaling, which causes the translocation of Rtg1p and Rtg3p, when cells contain intact RTG components (Table S1). The translocation of Rtg1p and Rtg3p can switch on the retrograde response, including the upregulation of CIT2 [16].

Sekito et al. 18 determined the nuclear concentration of Rtg1p, Rtg2p and Rtg3p relative to cytoplasmic levels via GFP labeling, RTG knockouts, and the comparison between $\rho^{+}$and $\rho^{0}$ cells. Therefore, the translocation event, existence of RTG components, and mitochondrial functionality can be summarized into a Boolean table, describing the system behavior of the RTG signaling pathway (Table 1) 18. The Boolean relation between mitochondrial damage (input) and the translocation of Rtg1p or Rtg3p can be further simplified by Karnaugh map (Fig 2a). 


\section{From Boolean model to an ordinary differential equation-based model}

To investigate the dynamics of mitochondria-nucleus communication, an ordinary differential equation-based model with two compartments (cytosol and nucleus) was developed to simulate RTG activation and translocation (Fig 1). Reactions of mitochondrial retrograde response were derived based on the law of mass action and Michaelis-Menten kinetics. There are total of 17 differential equations (S1.1) and 24 kinetic coefficients (Table S3) to describe the protein dynamics with different activation states or locations in the RTG signaling pathway .

The activation layer consists of Rtg2p, Mksp and Bmhp. This layer is constantly suppressed by Bmhp-Mksp heterodimer, and conveys the mitochondrial damage signal via Rtg2p activation. Later on, Rtg1p and Rtg3p form the modulation layer. Rtg3p contains nuclear localization sequence (NLS) and is poly-phosphorylated when the RTG pathway is inactive. In contrast, upon mitochondrial damage, Rtg3p is less phosphorylated and tends to translocate into the nucleus. The process of transcription factor translocation of modeled with simplified mass kinetics (Eq. 3) as previously described [59].

$$
T F_{c} \underset{k_{\text {out }}}{\stackrel{k_{\text {in }}}{\rightleftharpoons}} T F_{n}
$$

where $T F_{*}$ represents transcription factor, and the subscripts $*$ describes the location with $n$ representing nucleus and $c$ the cytosol. The compartmental model is further performed with the following equalities:

$$
\left[\begin{array}{l}
R t g 1_{c}^{\text {Total }} \\
R t g 1_{n}^{\text {Total }} \\
R t g 3_{c}^{\text {Total }} \\
R t g 3_{n}^{\text {Total }}
\end{array}\right]=\left[\begin{array}{cccccccccc}
1 & 0 & 0 & 0 & 0 & 0 & 1 & 1 & 0 & 0 \\
0 & 1 & 0 & 0 & 0 & 0 & 0 & 0 & 1 & 1 \\
0 & 0 & 1 & 0 & 1 & 0 & 1 & 1 & 0 & 0 \\
0 & 0 & 0 & 1 & 0 & 1 & 0 & 0 & 1 & 1
\end{array}\right]\left[\begin{array}{c}
R t g 1_{c} \\
R t g 1_{n} \\
R t g 3_{c}^{i} \\
R t g 3_{n}^{i} \\
R t g 3_{c}^{a} \\
R t g 3_{n}^{a} \\
R t g 1 / 3_{c}^{a} \\
R t g 1 / 3_{c}^{i} \\
R t g 1 / 3_{n}^{a} \\
R t g 1 / 3_{n}^{i}
\end{array}\right]
$$

where $\left[R \operatorname{Rtg} 1_{c}^{\text {Total }}\right]$ is defined as the total cytoplasmic concentration of Rtg1p 399 and heterodimers containing Rtg1p. The localization is labeled with cytosol $(c){ }_{400}$ and nucleus $(n)$ in subscript. $a$ and $i$ in superscript represent partial phosphorylation and hyper-phosphorylation forms of Rtg3p. The dephosphorylation process turns on the nucleus localization sequence (NLS) in Rtg3p [18].

\section{The differential equation-based model of RTG signaling}

A mathematical model was proposed to fulfilled the current understanding in signaling RTG pathway as summarized previously [18,32. The RTG pathway 
model was based on differential equations to explore the system dynamics. The 408 model was composed of biochemical reaction, Michaelis-Menten enzymatic kinetics, and Hill equation. The activation layer conveys the mitochondrial damage signal via Rtg2p activation. The modulation layer translocates Rtg3p and Rtg1p to nucleus to initiate RTG response.

1. Activation layer : Rtg2p and mitochondrial damage signal $(s)$.

where $\operatorname{Hill}\left(s, k_{s v}, k_{s d}, n\right)=k_{s v} \frac{s^{n}}{s^{n}+k_{s d}^{n}}$.

Competitive interaction between Rtg2p and Bmhp on Mksp.

$$
\begin{aligned}
& R t g 2_{c}^{a c t}+M k s \frac{k_{r m}}{\underset{k_{r m i}}{\rightleftharpoons}} R t g 2 M k s_{c} \\
& B m h+M k s \underset{k_{b m i}}{\stackrel{k_{b m}}{\rightleftharpoons}} B m h M k s
\end{aligned}
$$

2. Modulation layer: Translocation of Rtg3p, Rtg1p and their derivatives 18,33 .

The constant inhibition of Bmh/Mksp is described by

$$
\begin{aligned}
R t g 13_{c}^{a} & \stackrel{k_{I}^{13}+M M\left([\mathrm{BmhMks}], k_{v_{I}}^{13}, k_{d_{I}}^{13}\right)}{\longrightarrow} R \operatorname{tg} 13_{c}^{i} \\
\operatorname{Rtg} 3_{c}^{i} & \stackrel{k_{c_{A}}^{3}}{\rightleftharpoons k_{c_{I}}^{3}} \operatorname{Rtg} 3_{c}^{a} \\
\operatorname{Rtg} 3_{n}^{a} & \stackrel{k_{n_{I}}^{3}}{\longrightarrow} \operatorname{Rtg} 3_{n}^{i}
\end{aligned}
$$

where $M M\left([\mathrm{BmhMks}], k_{v_{I}}^{13}, k_{d_{I}}^{13}\right)=k_{v_{I}}^{13} \frac{[B m h M k s]}{[B m h M k s]+k_{d_{I}}^{13}}$

The formation of Rtg1/3

$$
\begin{gathered}
R t g 1_{c}+R t g 3_{c}^{a} \underset{k_{c_{I}}^{13}}{\stackrel{k_{c}^{13}}{\rightleftharpoons}} \operatorname{Rtg} 13_{c}^{a} \\
R t g 1_{c}+R \operatorname{Rg} 3_{c}^{i} \underset{k_{c_{I}}^{13}}{\stackrel{k_{c}^{13}}{\rightleftharpoons}} \operatorname{Rtg} 13_{c}^{i} \\
R t g 1_{n}+R t g 3_{n}^{a} \underset{k_{c_{I}}^{13}}{\stackrel{k_{n}^{13}}{\rightleftharpoons}} \operatorname{Rtg} 13_{n}^{a} \\
R t g 1_{n}+R t g 3_{n}^{i} \underset{k_{n_{I}}^{13}}{\stackrel{k_{n}^{13}}{\rightleftharpoons}} \operatorname{Rtg} 13_{n}^{i}
\end{gathered}
$$


Translocation of Rtg1/3p

$$
\begin{aligned}
& R t g 1_{c} \underset{k_{\text {out }}^{1}}{\stackrel{k_{\text {in }}^{1}}{\rightleftharpoons}} \operatorname{Rtg} 1_{n} \\
& \operatorname{Rtg} 3_{c}^{a} \underset{k_{a_{\text {out }}}^{3}}{\stackrel{k_{a_{\text {in }}}^{3}}{\rightleftharpoons}} \operatorname{Rtg} 3_{n}^{a} \\
& \operatorname{Rtg} 3_{c}^{i} \underset{k_{i_{\text {out }}}^{3}}{\stackrel{k_{i_{\text {in }}^{3}}^{3}}{\rightleftharpoons}} \operatorname{Rtg} 3_{n}^{i}
\end{aligned}
$$

To determine the relative concentrations of proteins in the RTG pathway, the ${ }_{421}$ expression level of RTG genes under normal and osmotic stress conditions (GEO access number: GSE59659) [35], and protein expression level obtained from Saccharomyces cerevisiae fusion library [34] were analyzed and used for determining the total concentration of RTG proteins(Fig $[$ S1).

The differential equations are described in Section S1.1. The parameter searching algorithm is in Section $\mathrm{S} 1.2$, and the final parameter set is listed in Table S3.

\section{Parameter searching with the qualitative data of protein translocation}

The parameters determined the steady-state behavior with respect to mitochondrial damage. A valid parameter set should make the model fulfill all conditions observed in $[18$ and 28 (Table 1). The parameter set was sampled in log-uniform with a fixed domain, and the relative amount of translocation kinetic coefficients is considered. To elaborate, the inward coefficient of nuclear Rtg3p with an active $\left(k_{a_{i n}}^{3}\right)$ was higher than the inactive one $\left(k_{i_{i n}}^{3}\right)$. Conversely, $k_{i_{\text {out }}}^{3}$ was higher than $k_{a_{\text {out }}}^{3}[59$.

The initial values of each component were sampled based on the RNA expression levels. The relative amount of protein under both normal and stress conditions was considered. The Boolean relations were tested by setting deleted proteins near zero, including modifications and complexes.

Once the parameters were sampled, the steady state of the model was derived by a dynamic solver, which provides an automatic switch between stiff and nonstiff algorithms when solving the differential equations. Furthermore, we chose Order $2 / 3$ L-Stable Rosenbrock-W method as the stiff solver, and Tsitouras 5/4 Runge-Kutta method for the nonstiff section [60].

The unstable solutions were discarded if the iteration exceeded $10^{6}$ with a deviation of derivatives beyond $10^{-8}$ in total. Further, the ratio of the nuclear concentration to cytoplasmic one was used to determine protein translocation. A nucleus-to-cytosol ratio larger than 1.5 was defined as a translocation event and vice versa. The translocation process under each deletion condition was compared with the Boolean model.

The differential equations were solved by DifferentialEquations.j1 (https://diffeq.sciml.ai/stable/), a open-source package for scientific computing 
implemented in the Julia programming language (https://julialang.org/). The parameter searching was performed on one machine with 30 Intel Xeon Processors in TWCC cloud service (https://www.twcc.ai/) for $10^{9}$ iterations.

\section{The analytical solution of the activation layer}

The acticvation layer contains three cytoplasmic proteins: Rtg2p, Bmh1/2p and Mks1p 13, 16, 21. A decline in mitochondrial membrane potential $\left(\Delta \Psi_{m}\right)$ leads to activation of Rtg2p, and interferences with the formation of Mks1p-Bmh1/2p complex, thereby impeding the nucleus accumulation of Rtg3p by inhibiting its partial dephosphorylation [16].

The reaction is summarized in the form of competitive binding with three components. Rtg2p and Bmhp (including Bmh1p and Bmh2p) are two ligands that compete for the Mks1p (Eq, 22). The input of the activation layer is the mitochondrial damage ( $s$ in Eq 22 ) which can be the decline of its $\Delta \Psi_{m}$, and further defined in the domain between 0 and 1 which represents the continuous state from healthy to the damaged. Further, the activation of Rtg2p induced by mitochondrial damage is modeled by a Hill equation. In the proposed model, the kinetic coefficients and total concentration of each protein are regarded as constants (Eqs. 25 and 28).

With the verified parameter set, the two-staged transition of Rtg2p activation induced by the mitochondrial damage signal was identified. The first stage of transition is a sigmoidal curve with the following steady region as the damage signal turns high, revealing the behavior of ultrasensitivity. After a certain value, the active form of Rtg2p increases in a convex trend with a slower slope compared to the first transition. Furthermore, by screening possible Hill coefficient of Rtg2p activation $\left(n^{s}\right)$, we found that the threshold of the first transition, rather than the stiffness of the curve, becoming larger when $n$ increases.

To further understand these phenomena, it is necessary to derive the analytical solution of steady states to understand the input-output relation of mitochondrial damage and the total concentration of Bmh/Mksp heterodimer. In the following paragraph, we used $\left[\mathrm{Rtg} 2_{c}^{a c t}\right],\left[\operatorname{Rtg} 2_{c}^{i n a}\right],[\mathrm{Bmh}]$ and $[\mathrm{Mks}]$ to represent their steady-state concentration.

The competitive binding of Bmhp and Rtg2p with Mksp is the source of the ultrasensitivity.

The modified competitive binding model from 61.

$$
\begin{aligned}
& \operatorname{Rtg} 2^{i n a} \stackrel{\frac{k_{s v} \frac{s^{n}}{k_{s v^{n}+s^{n}}}}{\rightleftharpoons k_{r i}}}{k_{r i}} \operatorname{Rt} 2^{a c t} \\
& M k s+R t g 2^{a c t} \underset{k_{I}^{r m}}{\stackrel{k_{r m}}{\stackrel{r_{m}}{r a}}} R t g 2 M k s \\
& M k s+B m h \underset{k_{b m i}}{\stackrel{k_{b m}}{\rightleftharpoons}} B m h M k s
\end{aligned}
$$


The dissociation coefficients are defined as

$$
\begin{aligned}
& K_{R}=\frac{\left[R t g 2^{a c t}\right][M k s]}{[R t g 2 M k s]}=\frac{k_{r m i}}{k_{r m}} \\
& K_{B}=\frac{[B m h][M k s]}{[B m h M k s]}=\frac{k_{b m i}}{k_{b m}} \\
& K_{T}=\frac{\left[R t g 2^{i n a}\right]}{\left[R t g 2^{a c t}\right]}=\frac{k_{r i}}{k_{s v} \frac{s^{n}}{k_{s v}+s^{n}}}
\end{aligned}
$$

where $K_{R}$ and $K_{B}$ are the dissociation coefficients for the Mksp's binding of Rtg2p ${ }^{a c t}$ and Bmhp. For the Rtg2p activation, We assume the input influcences the activation of Rtg2p $\mathrm{p}^{\text {ina }}$ in the manner of Hill formula (Eq.20 and Eq.25). To avoid zero division, we set the domain of input as $s \in(0,1]$.

The conservation rule requires that

$$
\begin{aligned}
& {[R t g 2]_{0}=\left[R t g 2^{i n a}\right]+\left[R t g 2^{a c t} M k s\right]+\left[R t g 2^{i n a}\right]} \\
& {[B m h]_{0}=[B m h]+[B m h M k s]} \\
& {[M k s]_{0}=[M k s]+\left[R t g 2^{a c t} M k s\right]+[B m h M k s]}
\end{aligned}
$$

where $\left[R t g 2_{c}^{a c t}\right]_{0},[B m h]_{0}$ and $\left[P_{0}\right]$ represent total concentration, and are assumed constants. Therefore, we can make $[B m h]$ and $\left[R t g 2^{a c t} M k s\right]$ into the dependent variables of $[M k s]$ and their total concentration.

$$
\begin{aligned}
{[B m h M k s] } & =\frac{[M k s][B m h]_{0}}{K_{B}+[M k s]} \\
{\left[R t g 2^{a c t} M k s\right] } & =\frac{\left[R t g 2_{c}^{a c t}\right]_{0}[M k s]}{\frac{K_{R}\left(1+K_{T}\right)}{K_{T}}+[M k s]}
\end{aligned}
$$

To derive the polynomial function of $[M k s]$, we can put Eq.29 and Eq.30 together:

$$
\begin{aligned}
{[M k s]_{0} } & =[M k s]+\left[R t g 2^{a c t} M k s\right]+[B m h M k s] \\
& =[M k s]+\frac{[M k s]\left[R t g 2_{c}^{a c t}\right]_{0}}{K_{R}\left(1+\frac{1}{K_{T}}\right)+[M k s]}+\frac{[M k s][B m h]_{0}}{K_{B}+[M k s]} \\
& =[M k s]+\underbrace{\frac{[M k s]\left[R t g 2_{c}^{a c t}\right]_{0}}{K_{R}^{\prime}+[M k s]}}_{=\left[R t g 2^{a c t} M k s\right]}+\underbrace{\frac{[M k s][B m h]_{0}}{K_{B}+[M k s]}}_{=[B m h M k s]}
\end{aligned}
$$

where $K_{R}^{\prime}=K_{R}\left(1+\frac{1}{K_{\triangle}}\right)$ is the dissociation coefficient influenced by the input coefficient $K_{T}(\mathrm{Eq} \cdot 25)$.

Finally, we can get the cubic polynomial equation of $[M k s]$ from Eq.31.

$$
[M k s]^{3}+a[M k s]^{2}+b[M k s]+c=0
$$


where

$$
\begin{aligned}
a & =K_{R}^{\prime}+K_{B}+\left[R t g 2_{c}^{a c t}\right]_{0}+[B m h]_{0}-[M k s]_{0} \\
b & =K_{B}\left(\left[R t g 2_{c}^{a c t}\right]_{0}-[M k s]_{0}\right)+K_{R}^{\prime}\left([B m h]_{0}-[M k s]_{0}\right)+K_{R}^{\prime} K_{B} \\
c & =-K_{R}^{\prime} K_{B}[M k s]_{0}
\end{aligned}
$$

The polynomial equation of $[M k s]$ can be solved by applying trigonometry 61,

The analytical solution of competitive ligand binding

$$
\begin{aligned}
{[M k s] } & =-\frac{a}{3}+\frac{2}{3} \sqrt{\left(a^{2}-3 b\right)} \cos \frac{\theta}{3} \\
{[R t g 2 M k s] } & =\frac{[R t g 2]_{0}\left(2 \sqrt{a^{2}-3 b} \cos (\theta / 3)-a\right)}{3 K_{R}+2 \sqrt{\left(a^{2}-3 b\right)} \cos (\theta / 3)-a} \\
{[B m h M k s] } & =\frac{[B m h]_{0}\left(2 \sqrt{a^{2}-3 b} \cos (\theta / 3)-a\right)}{3 K_{B}+2 \sqrt{\left(a^{2}-3 b\right)} \cos (\theta / 3)-a} \\
{[B m h] } & =[B m h]_{0}-[B m h M k s] \\
{\left[R t g 2^{a c t}\right] } & =\left([R t g 2]_{0}-[R t g 2 M k s]\right) \frac{1}{1+K_{T}} \\
{\left[R t g 2^{\text {ina }}\right] } & =\left([R t g 2]_{0}-[R t g 2 M k s]\right) \frac{K_{T}}{1+K_{T}}
\end{aligned}
$$

where $\theta=\cos ^{-1}\left(\frac{-2 a^{3}+9 a b-27 c}{2 \sqrt{\left(a^{2}-3 b\right)^{3}}}\right)$.

\section{Analysis ultrasensitivity with relative amplification approach}

The relative amplification approach is applied to quantify the local and global sensitivity by estimating the equivalent Hill coefficient [36]. When the response curve starts with a high basal level, this approach provides a more accurate approximation compared to the method using EC90:EC10 ratio [36, 57]. The response coefficient $\left(R_{\text {input }}^{\text {output }}, \mathrm{Eq} 39\right.$ is used to quantify the relative change in response to the stimulus.

$$
R_{\text {input }}^{\text {output }}=\frac{\text { input }}{\text { output }} \cdot \frac{d \text { output }}{d \text { input }}
$$

Furthermore, to circumvent the deviation from a high basal level, the activated fraction $(f, \mathrm{Eq} 40$ is defined as the activated ratio of output between the maximum and basal response 36 .

$$
f(\text { output })=\frac{\text { output }- \text { output }_{\text {basal }}}{\text { output }_{\max }-\text { output }_{\text {basal }}}
$$

where $f \in[0,1]$, output is the function of input. Besides, output $t_{\max }$ and output $_{\text {basal }}$ represents the maximum and minimum of outputs respectively, which makes activated fraction $(f)$ independent from the basal activation. 
The advantage of applying the response coefficient $(R)$ and activated fraction 521 $(f)$ is that the classical Hill equation can be converted to a linear equation $\left(\begin{array}{ll}\mathrm{Eq} & 522\end{array}\right.$ 41) 36 .

$$
\begin{gathered}
R=n \cdot(1-f) \\
n_{R}=\frac{\left|\int_{f_{L}}^{f_{H}} R_{f}^{\text {output }} d f\right|}{\left|\int_{f_{L}}^{f_{H}} R_{f_{M M}}^{\text {output }} d f\right|}
\end{gathered}
$$

where $f_{H}$ and $f_{L}$ are the maximum and minimum of activation fraction in a given region.

\section{Frequency response of RTG signaling}

To measure the RTG response to the dynamical input, the original ODE model is converted into non-autonomous differential equations with mitochondrial damage signal $(s)$ as input (Eq,43).

$$
\frac{d[\text { Output }]}{d t}=A f\left(s(t) ; u_{s}^{p}, p\right)
$$

where $A$ is the output map

Furthermore, the sinusoidal function with frequency between $10^{-3} \mathrm{~Hz}$ and 1 $\mathrm{Hz}$ was used to conduct the frequency analysis to reveal the break point in Bode diagram. In this study, we focus on the concentrations of Bmh/Mksp heterodimer and Rtg1/3p nucleus accumulation in response to the sinusoidal input.

The nonautonomous model is solved by Rosenbrock 23 with automatic switching for Tsit5 solver. The Rosenbrock23 is a stiff ODE solver which can deal with more general situation than Tsit5, a non-stiff solver, but with lower speed [60]. By the aid of automatic switching stiff/non-stiff method of DifferentialEquations.jl [60], the simulation process can be significantly sped up.

\section{Stochastic simulation of Rtg1/3p translocation in response to mitochondrial damage}

The stochastic model is modified from the ODEs with the theory of Chemical Langevin Equation (CLE) 64]. The derivative of nucleus Rtg1/3p is composed of the drifting term from the deterministic model, and the diffusion term caused by the Brownian motion. The process is described with the stochastic differential equation in Eq 44 .

$$
d\left[R \operatorname{Rt} 13_{n}\right]_{t}=\underbrace{\sum_{r=1}^{R} S_{r} f_{r}\left(\left[\operatorname{Rtg} 13_{n}\right]_{t}\right) d t}_{\text {Drifting }}+\underbrace{\sqrt{\sum_{r=1}^{R} S_{r}^{2} f_{r}\left(\left[\operatorname{Rtg} 13_{n}\right]_{t}\right)} d W}_{\text {Diffusion }}
$$

where $f_{r}$ is the propensity of $r^{t h}$ reaction, $S_{r}$ the net change of the reaction $r, \quad{ }^{548}$ and $d W$ represents the Wiener process [65]. 
The CLE was solved using the Euler-Maruyama method with adaptive time stepping, and simulated for 100 seconds. Further, the time series simulation was used to derive the likelihood of the output state with kernel density estimation as illustrated in Fig 5 .

\section{References}

1. Osellame LD, Blacker TS, Duchen MR. Cellular and molecular mechanisms of mitochondrial function. Best Practice and Research: Clinical Endocrinology and Metabolism. 2012;26(6):711-723. doi:10.1016/j.beem.2012.05.003.

550 551 552 553

2. Nicholls DG, Budd SL. Mitochondria and neuronal survival. Physiological Reviews. 2000;80(1):315-360. doi:10.1152/physrev.2000.80.1.315.

3. Friedman JR, Nunnari J. Mitochondrial form and function. Nature. 2014;505(7483):335-343. doi:10.1038/nature12985.

4. Mottis A, Herzig S, Auwerx J. Mitocellular communication: Shaping health and disease. 2019;366(6467):827-832. doi:10.1126/science.aax3768.

5. Liu Z, Butow Ra. Mitochondrial retrograde signaling. Annual review of genetics. 2006;40:159-185. doi:10.1146/annurev.genet.40.110405.090613.

6. Ždralević M, Guaragnella N, Antonacci L, Marra E, Giannattasio S. Yeast as a Tool to Study Signaling Pathways in Mitochondrial Stress Response and Cytoprotection. The Scientific World Journal. 2012;2012:1-10. doi:10.1100/2012/912147.

7. Aon MA, Bhatt N, Cortassa SC. Mitochondrial and cellular mechanisms for managing lipid excess. Frontiers in physiology. 2014;5:282.

8. King N. Amino acids and the mitochondria. In: Mitochondria. Springer; 2007. p. 151-166.

9. Knorre D, Sokolov S, Zyrina A, Severin F. How do yeast sense mitochondrial dysfunction? Microbial Cell. 2016;3(11):401-408. doi:10.15698/mic2016.11.537.

10. Gabaldón T, Huynen MA. Shaping the mitochondrial proteome. Biochimica et Biophysica Acta - Bioenergetics. 2004;1659(2-3):212-220. doi:10.1016/j.bbabio.2004.07.011.

11. Malina C, Larsson C, Nielsen J. Yeast mitochondria: An overview of mitochondrial biology and the potential of mitochondrial systems biology. FEMS Yeast Research. 2018;18(5):1-17. doi:10.1093/femsyr/foy040.

12. Da Cunha FM, Torelli NQ, Kowaltowski AJ. Mitochondrial Retrograde Signaling: Triggers, Pathways, and Outcomes. Oxidative Medicine and Cellular Longevity. 2015;2015. doi:10.1155/2015/482582. 
13. Butow RA, Avadhani NG. Mitochondrial signaling: The retrograde 587 response. Molecular Cell. 2004;14(1):1-15. doi:10.1016/S1097-2765(04)00179-0.

14. Cagin U, Enriquez JA. The complex crosstalk between mitochondria and the nucleus: What goes in between? International Journal of Biochemistry and Cell Biology. 2015;63(February):10-15. doi:10.1016/j.biocel.2015.01.026.

15. Chelstowska A, Liu Z, Jia Y, Amberg D, Butow RA. Signalling between mitochondria and the nucleus regulates the expression of a new D-lactate dehydrogenase activity in yeast. Yeast. 1999;15(13):1377-1391. doi:10.1002/(SICI)1097-0061(19990930)15:13<1377::AIDYEA473>3.0.CO;2-0.

16. Jazwinski SM, Kriete A. The yeast retrograde response as a model of intracellular signaling of mitochondrial dysfunction. Frontiers in Physiology. 2012;3 MAY(May):1-12. doi:10.3389/fphys.2012.00139.

17. Liao X, Butow RA. RTG1 and RTG2: Two yeast genes required for a novel path of communication from mitochondria to the nucleus. Cell. 1993;72(1):61-71. doi:10.1016/0092-8674(93)90050-Z.

18. Sekito T, Thornton J, Butow RA. Mitochondria-to-nuclear signaling is regulated by the subcellular localization of the transcription factors Rtg1p and Rtg3p. Molecular biology of the cell. 2000;11(6):2103-15. doi:10.1091/mbc.11.6.2103.

19. Epstein CB, Waddle JA, Hale IV W, Davé V, Thornton J, Macatee TL, et al. Genome-wide responses to mitochondrial dysfunction. Molecular Biology of the Cell. 2001;12(2):297-308. doi:10.1091/mbc.12.2.297.

20. Liu Z, Butow Ra. A transcriptional switch in the expression of yeast tricarboxylic acid cycle genes in response to a reduction or loss of respiratory function. Molecular and cellular biology. 1999;19(10):6720-8. doi:6720-6728.

21. Ferreira JR, Spírek M, Liu Z, Butow RA. Interaction between Rtg2p and Mks1p in the regulation of the RTG pathway of Saccharomyces cerevisiae. Gene. 2005;354(1-2 SPEC. ISS.):2-8. doi:10.1016/j.gene.2005.03.048.

22. Jazwinski SM. The retrograde response: When mitochondrial quality control is not enough. Biochimica et Biophysica Acta - Molecular Cell Research. 2013;1833(2):400-409. doi:10.1016/j.bbamcr.2012.02.010.

23. Hashim Z, Mukai Y, Bamba T, Fukusaki E. Metabolic profiling of retrograde pathway transcription factors rtg1 and rtg3 knockout yeast. Metabolites. 2014;4(3):580-98. doi:10.3390/metabo4030580. 
24. Rothermel BA, Thornton JL, Butow RA. Rtg3p, a basic helix-loop-helix/leucine zipper protein that functions in mitochondrial-induced changes in gene expression, contains independent activation domains. Journal of Biological Chemistry. 1997;272(32):19801-19807. doi:10.1074/jbc.272.32.19801.

25. Cover TM, Thomas JA. Elements of Information Theory. Wiley; 2005.

26. Fatima Z, Kumar B, Rehman S, Hameed S. Retrograde signaling: A novel antifungal drug target. In: New and Future Developments in Microbial Biotechnology and Bioengineering. Elsevier; 2020. p. 219-226.

27. Trendeleva T, Zvyagilskaya R. Retrograde Signaling as a Mechanism of Yeast Adaptation to Unfavorable Factors. Biochemistry (Moscow). 2018;83(2):98-106. doi:10.1134/S0006297918020025.

28. Liu Z, Sekito T, Epstein CB, Butow RA. RTG-dependent mitochondria to nucleus signaling is negatively regulated by the seven WD-repeat protein Lst8p. EMBO Journal. 2002;20(24):7209-7219. doi:10.1093/emboj/20.24.7209.

29. Hans S, Fatima Z, Hameed S. Retrograde signaling disruption influences ABC superfamily transporter, ergosterol and chitin levels along with biofilm formation in Candida albicans. Journal de Mycologie Medicale. 2019;29(3):210-218. doi:10.1016/j.mycmed.2019.07.003.

30. Moye-Rowley WS. Retrograde regulation of multidrug resistance in Saccharomyces cerevisiae. In: Gene. vol. 354. Gene; 2005. p. 15-21.

31. Yan H, Zhao Y, Jiang L. The putative transcription factor CaRtg3 is involved in tolerance to cations and antifungal drugs as well as serum-induced filamentation in Candida albicans. FEMS yeast research. 2014;14(4):614-623. doi:10.1371/journal.pone.0203079.

32. Sekito T, Liu Z, Thornton J, Butow RA. RTG-dependent mitochondria-to-nucleus signaling is regulated by MKS1 and is linked to formation of yeast prion [URE3]. Molecular biology of the cell. 2002;13(3):795-804. doi:10.1091/mbc.01-09-0473.

33. Dilova I, Aronova S, Chen JCY, Powers T. Tor signaling and nutrient-based signals converge on Mks1p phosphorylation to regulate expression of Rtg1p Rtg3p-dependent target genes. Journal of Biological Chemistry. 2004;279(45):46527-46535. doi:10.1074/jbc.M409012200.

34. Ghaemmaghami S, Huh WK, Bower K, Howson RW, Belle A, Dephoure $\mathrm{N}$, et al. Global analysis of protein expression in yeast. Nature. 2003;425(6959):737-741.

35. Gomar-Alba M, Morcillo-Parra MA, Olmo MLD. Response of yeast cells to high glucose involves molecular and physiological differences when 
compared to other osmostress conditions. FEMS Yeast Research. 2015;15(5):fov039. doi:10.1093/femsyr/fov039.

36. Legewie S, Blüthgen N, Herzel H. Quantitative analysis of ultrasensitive responses. FEBS Journal. 2005;272(16):4071-4079. doi:10.1111/j.1742-4658.2005.04818.x.

37. Alon U. An introduction to systems biology: design principles of biological circuits. CRC press; 2019. p. 37-54.

38. Iglesias PA, Ingalls BP. Control Theory and Systems Biology. MIT Press; 2009.

39. Tsimring, L.S., Noise in Biology Reports on Progress in Physics. 2014 Jan 20;77(2):026601.

40. Buchler NE, Louis M. Molecular titration and ultrasensitivity in regulatory networks. Journal of molecular biology. 2008;384(5):1106-1119. doi:10.1016/j.jmb.2008.09.079.

41. Haney S, Bardwell L, Nie Q. Ultrasensitive responses and specificity in cell signaling. BMC systems biology. 2010;4(1):119. doi:10.1186/1752-0509-4-119.

42. Thattai M, van Oudenaarden A. Attenuation of noise in ultrasensitive signaling cascades. Biophysical journal. 2002;82(6):2943-2950. doi:10.1016/S0006-3495(02)75635-X.

43. Butow Ra. Cellular responses to mitochondrial dysfunction: it's not always downhill. Cell death and differentiation. 2002;9(10):1043-5. doi:10.1038/sj.cdd.4401083.

44. Lloyd D, Eshantha L, Salgado J, Turner MP, Murray DB. Respiratory oscillations in yeast: clock-driven mitochondrial cycles of energization. FEBS letters. 2002;519(1-3):41-44.

45. Lloyd D, Lemar KM, Salgado LEJ, Gould TM, Murray DB. Respiratory oscillations in yeast: mitochondrial reactive oxygen species, apoptosis and time; a hypothesis. FEMS yeast research. 2003;3(4):333-339.

doi:10.1016/S1567-1356(03)00071-0.

46. Murray DB, Roller S, Kuriyama H, Lloyd D. Clock control of ultradian respiratory oscillation found during yeast continuous culture. Journal of Bacteriology. 2001;183(24):7253-7259. doi:10.1128/JB.183.24.7253-7259.2001.

47. Heart E, Yaney GC, Corkey RF, Schultz V, Luc E, Liu L, et al. Ca2+, NAD (P) $\mathrm{H}$ and membrane potential changes in pancreatic $\beta$-cells by methyl succinate: comparison with glucose. Biochemical Journal. 2007;403(1):197-205. doi:10.1042/BJ20061209. 
48. Chen WW, Freinkman E, Wang T, Birsoy K, Sabatini DM. Absolute 702 Quantification of Matrix Metabolites Reveals the Dynamics of Mitochondrial Metabolism. Cell. 2016; p. 1324-1337. doi:10.1016/j.cell.2016.07.040.

49. Christian BE, Shadel GS. Aging: It's SIRTainly possible to restore mitochondrial dysfunction. Current Biology. 2014;24(5):R206-R208. doi:10.1016/j.cub.2014.01.027.

50. Miceli MV, Jiang JC, Tiwari A, Rodriguez-Quiñones JF, Jazwinski SM. Loss of mitochondrial membrane potential triggers the retrograde response extending yeast replicative lifespan. Frontiers in Genetics. 2012;2(JAN):1-11. doi:10.3389/fgene.2011.00102.

51. Nyström T. Aging: Filtering out bad mitochondria. Current Biology. 2013;23(23):1037-1039. doi:10.1016/j.cub.2013.10.049.

52. Whelan SP, Zuckerbraun BS. Mitochondrial Signaling: Forwards, Backwards, and In Between. Oxidative Medicine and Cellular Longevity. 2013;2013:1-10. doi:10.1155/2013/351613.

53. Badiee P, Hashemizadeh Z. Opportunistic invasive fungal infections: diagnosis \& clinical management. The Indian journal of medical research. 2014;139(2):195-204.

54. Jastrzebowska K, Gabriel I. Inhibitors of amino acids biosynthesis as antifungal agents. Amino acids. 2015;47(2):227-249. doi:10.1007/s00726-014-1873-1.

55. Pasko MT, Piscitelli SC, Van Slooten AD. Fluconazole: a new triazole antifungal agent. Dicp. 1990;24(9):860-867.

56. Singh S, Fatima Z, Ahmad K, Hameed S. Fungicidal action of geraniol against Candida albicans is potentiated by abrogated CaCdr1p drug efflux and fluconazole synergism. PloS one. 2018;13(8):e0203079. doi:10.1371/journal.pone.0203079.

57. Ferrell JE, Ha SH. Ultrasensitivity part I: Michaelian responses and zero-order ultrasensitivity; 2014.

58. Delmans M, Hemberg M. Discrete distributional differential expression (D3E) - a tool for gene expression analysis of single-cell RNA-seq data. BMC Bioinformatics. 2016;17(1):110. doi:10.1186/s12859-016-0944-6.

59. Hao N, Budnik BA, Gunawardena J, O'Shea EK. Tunable signal processing through modular control of transcription factor translocation. Science. 2013;339(6118):460-464. doi:10.1126/science.1227299.

60. Rackauckas C, Nie Q. DifferentialEquations.jl - A Performant and Feature-Rich Ecosystem for Solving Differential Equations in Julia. Journal of open research software. 2017;5. doi:10.5334/jors.151. 
61. Wang ZX. An exact mathematical expression for describing competitive 741 binding of two different ligands to a protein molecule. FEBS Letters. $\quad 742$ 1995;360(2):111-114. doi:10.1016/0014-5793(95)00062-E.

62. Harley H. McAdams and Adam Arkin Stochastic mechanisms in gene expression Proceedings of the National Academy of Sciences. 1997; 94.3: 814-819.

63. Cheong, R., Rhee, A., Wang, C. J., Nemenman, I., Levchenko, A. Information transduction capacity of noisy biochemical signaling networks science, 2011, 334.6054: 354-358.

64. Gillespie DT. Chemical Langevin equation. Journal of Chemical Physics. 750 2000;113(1):297-306. doi:10.1063/1.481811.

65. Schnoerr D, Sanguinetti G, Grima R. The complex chemical Langevin equation. The Journal of chemical physics. 2014;141(2):07B606_1. 
(a)

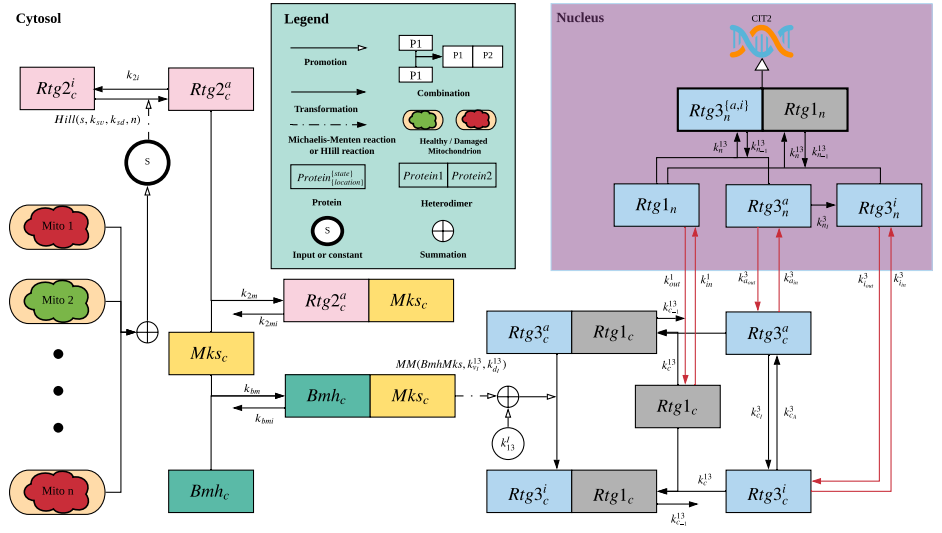

Activation Layer

Modulation Layer

(b)
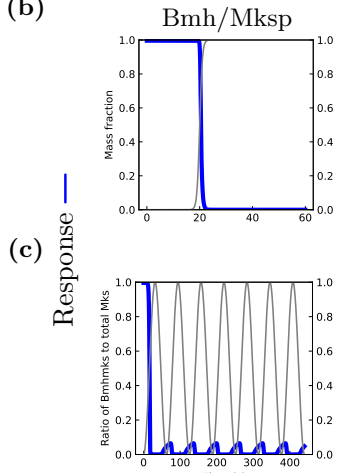
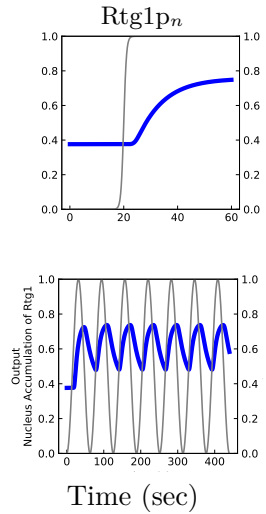
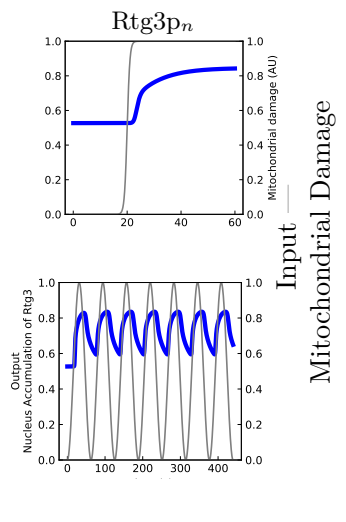


\section{Rtg3-GFP}

Existance(Rtg3p)

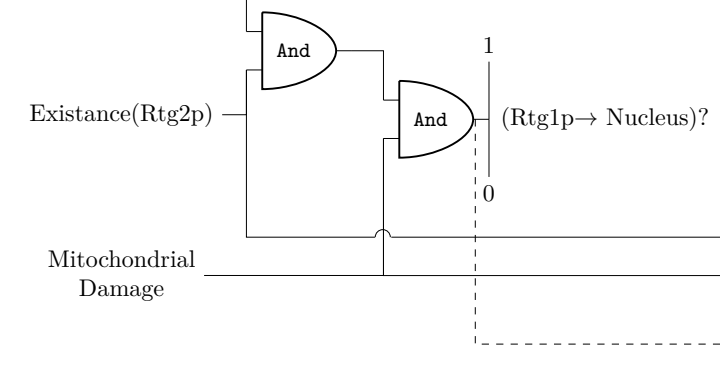

Existance(Rtg1p) - Not $>$

\section{(b)}

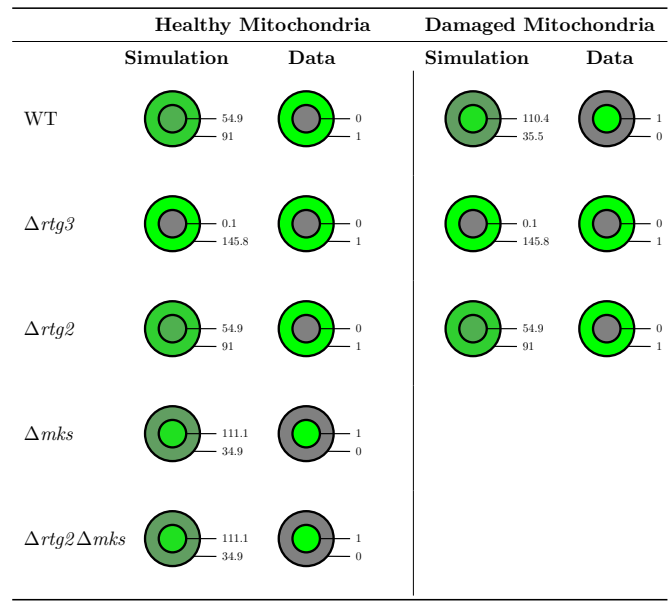

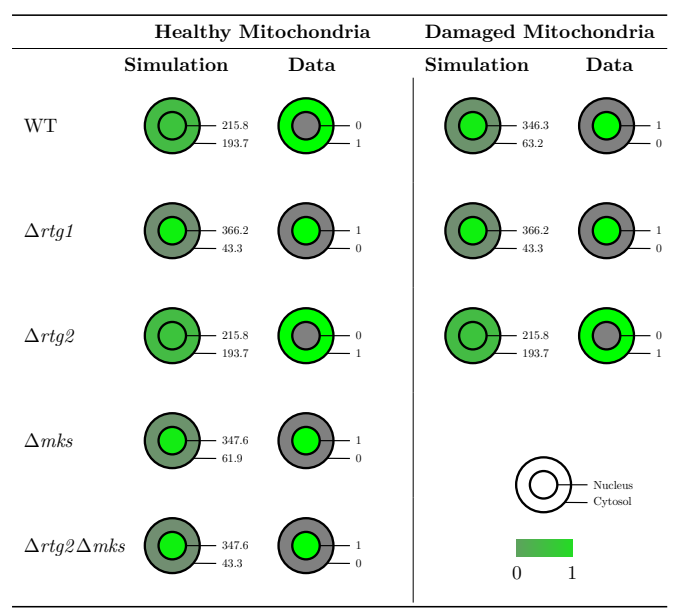


(a)

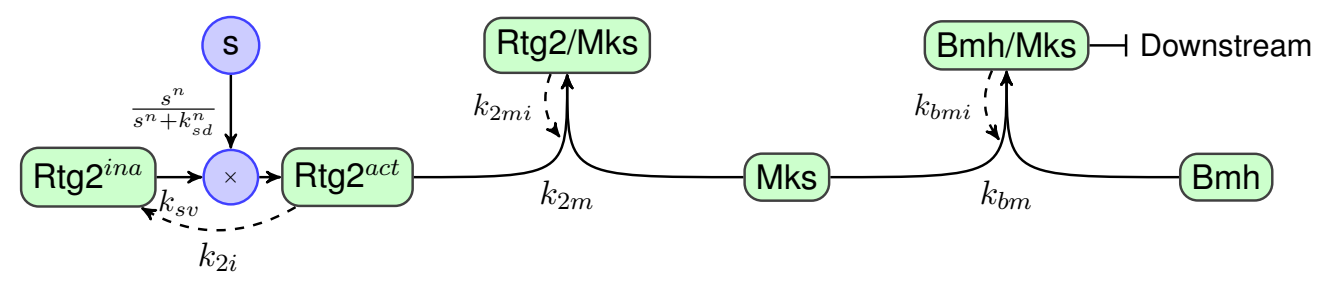

(b)

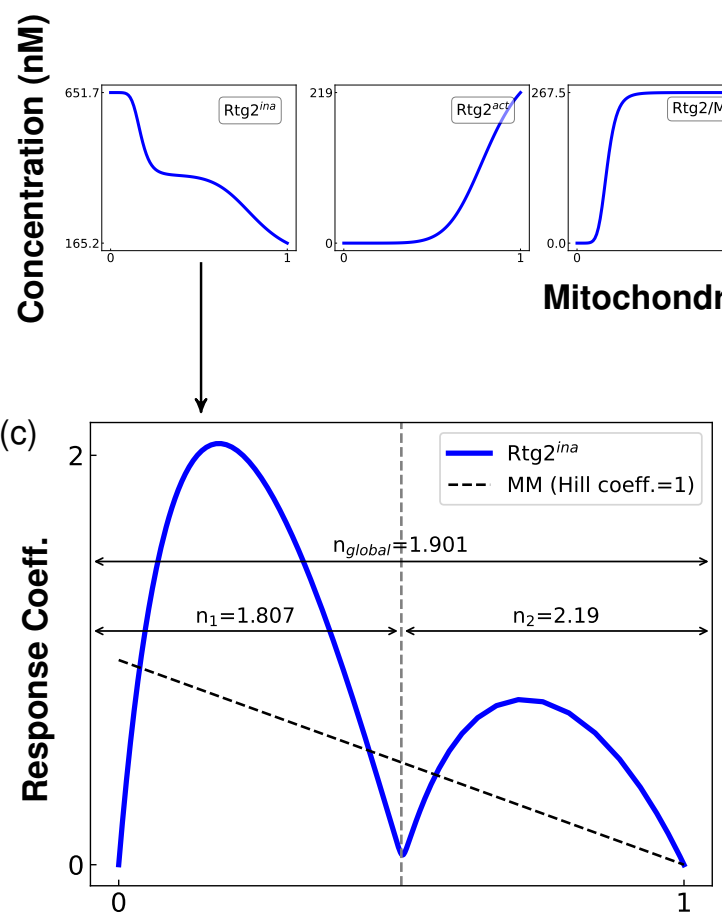

Activation Fraction

\section{Reaction Network}

$$
R \operatorname{Rg} 2^{i n a} \underset{k_{s v} \frac{s^{n}}{k_{s v^{n}+s^{n}}}}{\rightleftharpoons k_{r i}} R t g 2^{a c t}
$$

$M k s+R t g 2^{a c t} \underset{k_{I}^{r m}}{\stackrel{k_{r m}}{\rightleftharpoons}} R t g 2 M k s$

$$
M k s+B m h \underset{k_{b m i}}{\stackrel{k_{b m}}{\rightleftharpoons}} B m h M k s
$$

\section{Steady State Analysis}

$$
[M k s]=-\frac{a}{3}+\frac{2}{3} \sqrt{\left(a^{2}-3 b\right)} \cos \frac{\theta}{3}
$$

$[R t g 2 M k s]=\frac{[R t g 2]_{0}\left(2 \sqrt{a^{2}-3 b} \cos (\theta / 3)-a\right)}{3 K_{R}+2 \sqrt{\left(a^{2}-3 b\right)} \cos (\theta / 3)-a}$

$[B m h M k s]=\frac{[B m h]_{0}\left(2 \sqrt{a^{2}-3 b} \cos (\theta / 3)-a\right)}{3 K_{B}+2 \sqrt{\left(a^{2}-3 b\right)} \cos (\theta / 3)-a}$ 
(a)

Inputs

(Mitochondrial damage)

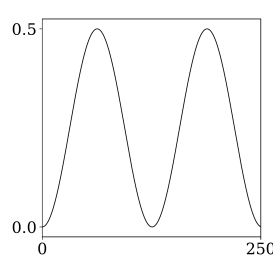

Source A

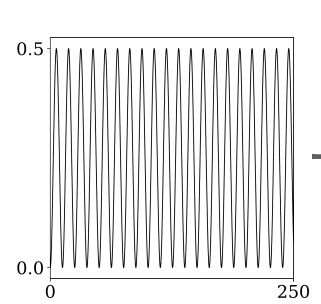

Source B
Combined signal $(A+B)$

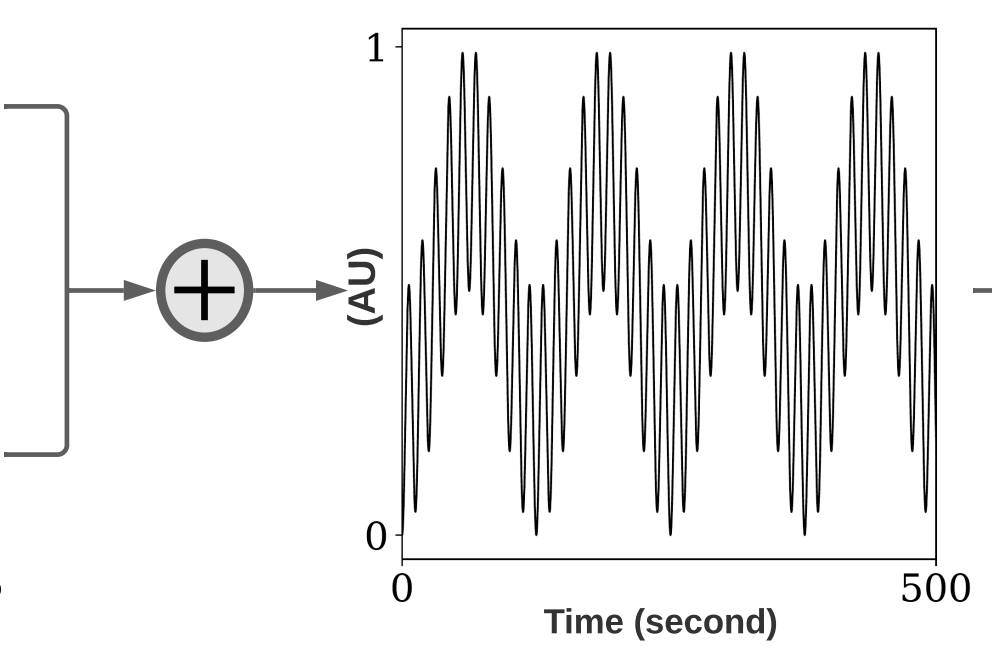

(b)

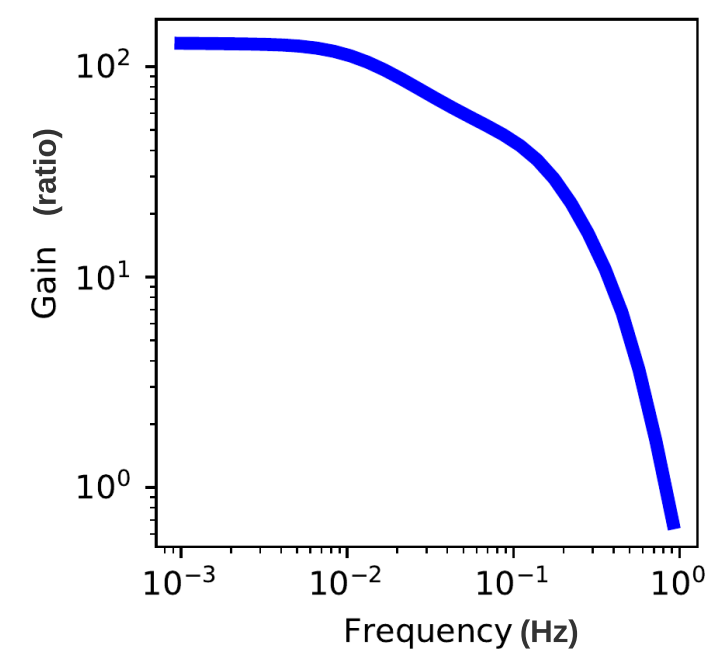

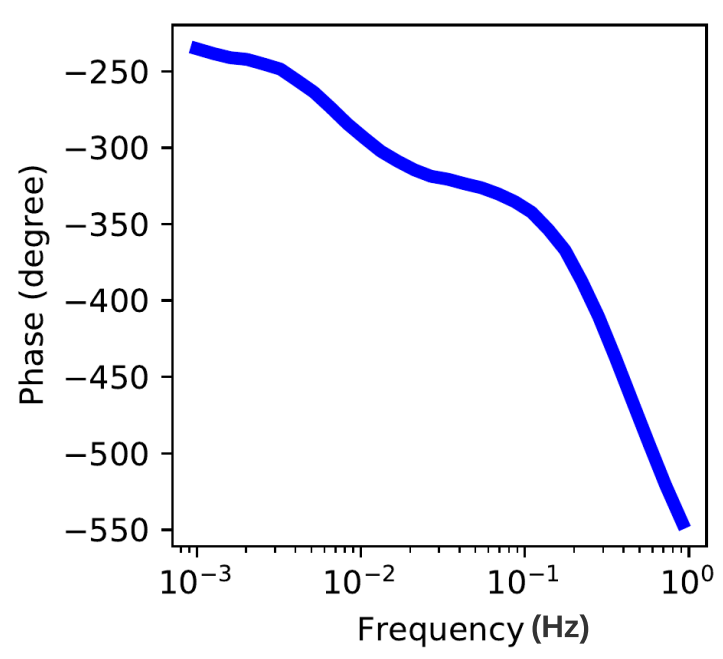

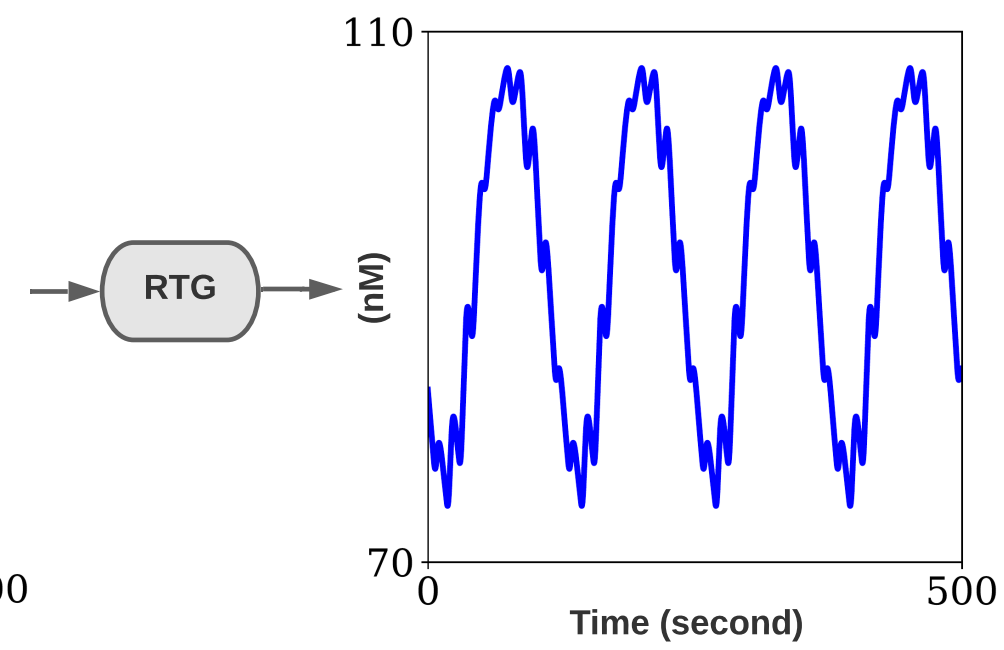

Output

(Nucleus Rtg1/3p)

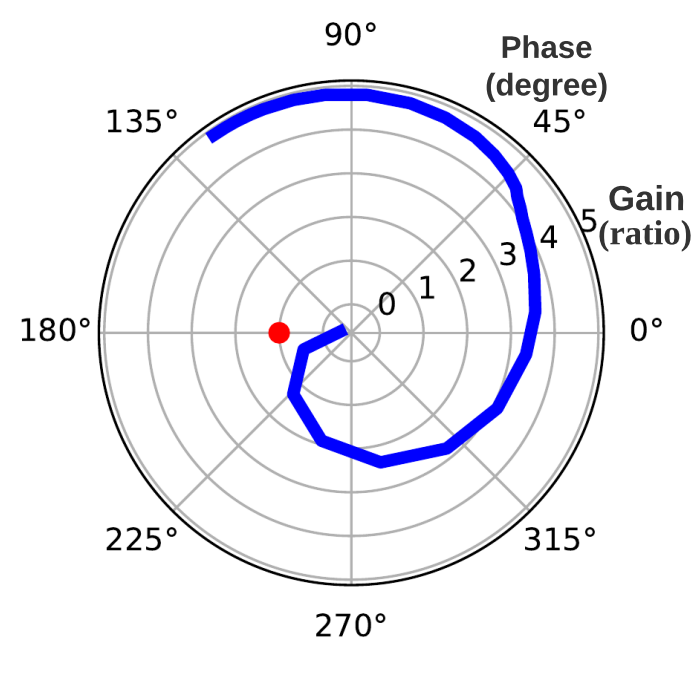


(a)
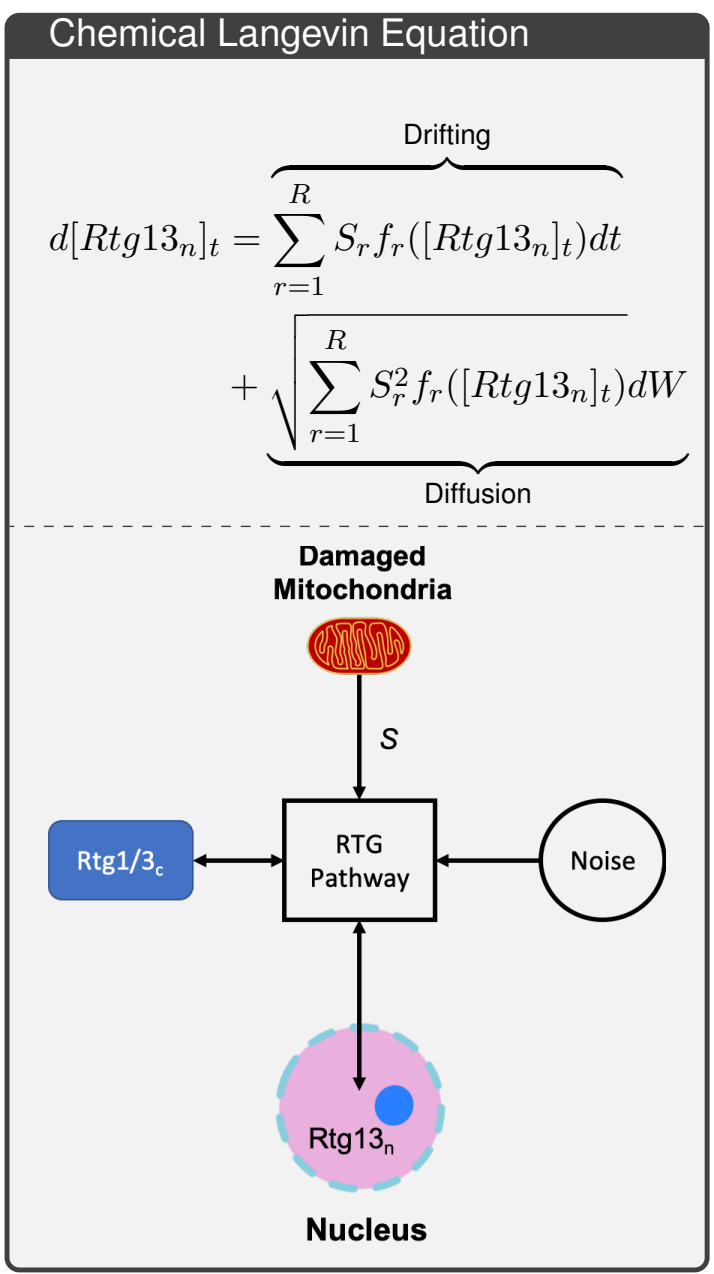

(b)

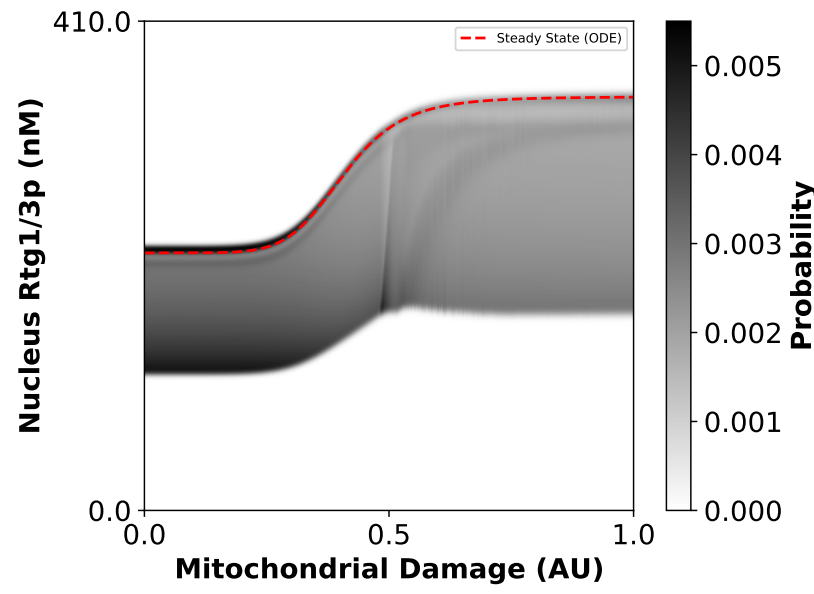

(c)

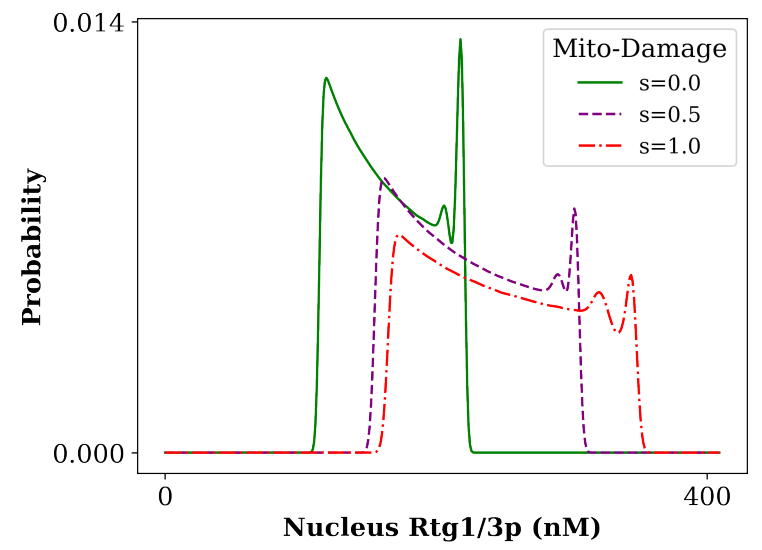


bioRxiv preprint doi: https://doi.org/10.1101/2021.03.27.437239; this version posted May 15, 2021. The copyright holder for this preprint (whic

(a) was not certified by peer review) is the author/funder. All rights reserved. No reuse allowed without permission.

\section{Molecular Circuit}

\section{Activation Layer Modulation Layer}

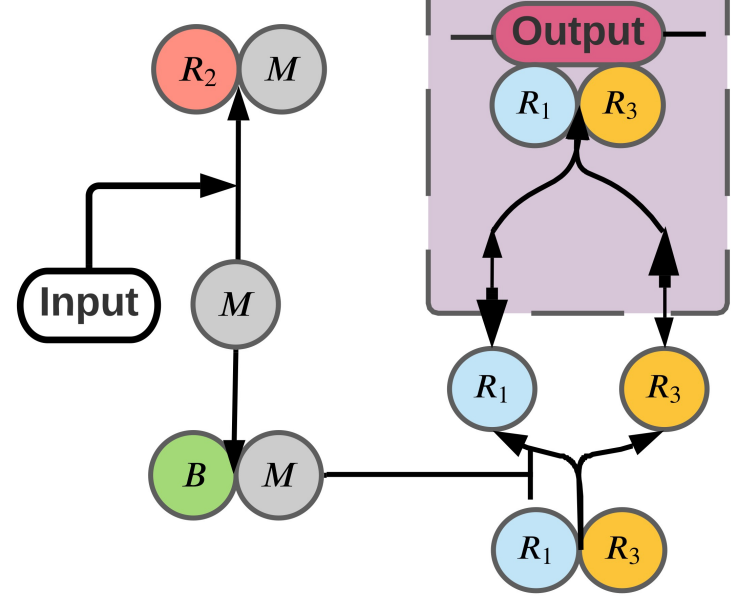

(b)

\section{Equivalent Circuit}

\section{Low-pass filter}

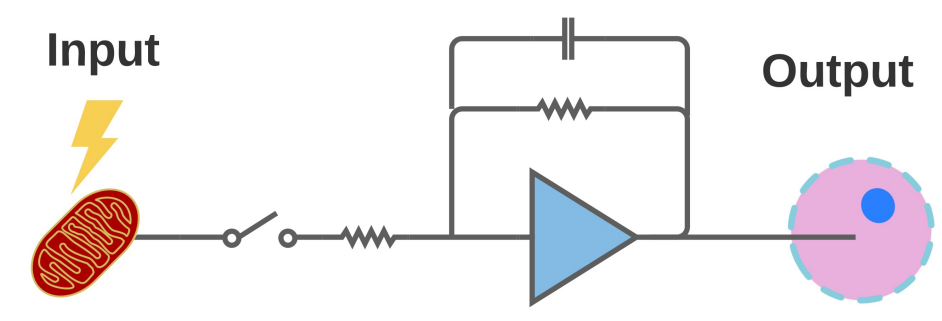

Mitochondrial

Receiver 